\title{
Fake News et conception catholique de la vérité médiatique
}

Fake News and the Catholic Vision of Media Truth

\section{David Douyère}

\section{(2) OpenEdition}

\section{Journals}

Édition électronique

URL : https://journals.openedition.org/edc/9319

DOI : 10.4000/edc.9319

ISSN : 2101-0366

Éditeur

Université de Lille

Édition imprimée

Date de publication : 15 décembre 2019

Pagination : 103-120

ISBN : 978-2-917562-22-2

ISSN : $1270-6841$

Référence électronique

David Douyère, «Fake News et conception catholique de la vérité médiatique », Études de

communication [En ligne], 53 | 2019, mis en ligne le 01 janvier 2022, consulté le 06 janvier 2022. URL

http://journals.openedition.org/edc/9319 ; DOI : https://doi.org/10.4000/edc.9319 
Fake News et conception catholique de la vérité médiatique

Fake News and the Catholic Vision of Media Truth 
Dans le $52^{\mathrm{e}}$ message pour la journée mondiale des communications sociales diffusé par le Vatican, "La vérité vous rendra libres, Fake news et journalisme de paix " (2018), le pape catholique François voit dans la fausse information l'œuvre du diable, comme dans l'action du serpent au paradis terrestre : les fake news déstructurent selon lui l'humain et la société. Or, en théologie catholique, le Christ est vérité. II propose donc une prière contre les fake news et invite les journalistes à faire œuvre de vérité, dans le prolongement d'une injonction pontificale présente dès les années 1930. Cette position atteste d'une appropriation singulière de la thématique des fake news en régime chrétien catholique et forme l'occasion d'une ouverture sur l'espace public et médiatique.

Mots-clés : vérité, médias, journalisme, diable, fake news, catholicisme, pape.
In his message for the 2018 World Communications Day entitled "The truth will set you free', fake news and journalism for peace", Pope Francis denounces fake news as the work of the Devil and suggests that, like the snake in the garden of Eden, fake news destabilizes humanity and society. The Pope offers a prayer against fake news and invites journalists to work for truth. This message can be seen as the extension of a papal request dating back to the 1930s. This position reveals a particular use of fake news in a Catholic perspective and it provides the Church with an occasion to extend its discourse into the public and media spheres.

Keywords: truth, media, journalism, devil, fake news, pope, catholicism. 


\section{Introduction}

Les fake news se trouvent, dans I'allocution du Pape François pour la journée mondiale des communications sociales de 2018, La vérité vous rendra libres, Fake news et journalisme de paix, pensées dans une dimension théologique comme une action maligne qui prend sa source au jardin d'Éden, quand Adam et Ėve y cèdent au "serpent rusé [...], celui qui, au commencement de I'humanité, est devenu l'auteur de la première "fake news" (cf. Gn 3,1-15), qui a conduit aux conséquences tragiques du péché, mises en acte ensuite [...] dans d'autres formes innombrables du mal contre Dieu, le prochain, la société et la création " (Pape François, 2018). Les fake news, dans cette perspective chrétienne catholique, "c'est la dynamique du mal qui se présente toujours comme un bien facilement accessible » (Viganò, dans Menichetti, 2018). Et le pape de proposer une prière contre les fake news ("Là où il y a la fausseté, que nous apportions la vérité $»^{1}$, Pape François, 2018). La présence du terme fake news, dans le discours pontifical et ecclésial, depuis quelques années, ne cesse en effet de surprendre, tout en se posant comme une évidence : discours du Pape François (Bevilacqua, 2018), conférence à Lourdes (2017), position de la Conférence des évêques de France (Turini, 2017 ; Douyère, 2017).

Dans la dynamique d'une exploration des « imaginaires » de la notion de fake news et de la circulation culturelle de cette dernière, nous proposons, par cet article, de mettre en lumière la conception catholique indigène et singulière des fake news, qui en fait pour ceux qui la portent l'occasion d'une évangélisation (Douyère, 2015) adaptée au temps. Cette appropriation singulière et opportuniste, captant le langage de l'époque, de la notion informationnelle et journalistique issue de la culture américaine (qui serait due au journaliste canadien Craig Silverman, en 2017 ; Thibault, 2018), qui désigne la désinformation (Sauvageau et al., 2018, 3), ou dévalorise, annihile l'information perçue comme gênante (Arêas, 2018), relève bien évidemment d'une trivialisation (Jeanneret, 2014) de la notion. Elle comporte une visée pour la société contemporaine : faire du faux l'occasion de l'entrée dans le vrai (religieux chrétien). La notion de fake news constitue donc un point d'entrée et de conversion de la société contemporaine, pour l'Église catholique. Par ailleurs, l'institution se pose ainsi comme porteuse d'une parole rectificatrice pour le monde (associée à la critique de l'individualisme, des attaques contre "la vie », du capitalisme ou du libéralisme à outrance, etc.), à portée parénétique, d'exhortation morale (Dufour, 2014). L'insistance portée sur la vérité dans la critique des fake news atteste en effet d'un conflit de sens avec le monde, qui fait partie du positionnement du christianisme, dans la tradition paulinienne (en référence à Paul de Tarse, dit saint Paul). 
Nous proposons ici, de façon singulière et inhabituelle, d'analyser un seul texte, le $52^{\mathrm{e}}$ message pour la journée mondiale des communications sociales, porté par le Pape François, La vérité vous rendra libres, Fake news et journalisme de paix (2018) pour comprendre cette doctrine et cette liaison entre la thématique informationnelle médiatique (et numérique) et la proposition théologique et morale catholique. Ce texte de référence pour l'Église catholique est en effet destiné à être lu et commenté par l'ensemble des diocèses et des paroisses dans le cadre de la " communication sociale " (Douyère, 2010) de cette dernière. II s'agit en effet ici d'expliciter, notamment par la présentation de citations de textes pontificaux, une position de ce que d'aucuns appelleraient des " nomothètes », des acteurs en position d'instituer une loi, une référence doctrinale, et du discours qui met ces principes en circulation, dans un contexte où la religion est d'emblée et pleinement communicationnelle (Pace, 2011).

La présente recherche s'inscrit à cet égard, pour examiner le discours catholique pontifical sur les fake news, dans un double ancrage scientifique : une recherche menée depuis plusieurs années sur les raisons et les modalités de la communication chrétienne catholique (Douyère, 2018) et une recherche collective $^{2}$ engagée sur la vérification informationnelle, le fact checking et les supposées fake news, avec des chercheurs travaillant plus spécifiquement sur le journalisme (Bigot, 2019 ; Bigot et Nicey, à paraître). Cet article se situe donc à l'interface des deux perspectives, qu'il se propose de nouer un instant. Après avoir examiné la relation de l'Église catholique à la notion de fake news, qui constitue une opportunité d'affirmer un rapport singulier à la vérité, nous situerons en premier lieu cette position dans le contexte des discours pontificaux sur le journalisme, en donnant quelques exemples succincts de ces derniers, puis étudierons l'abord de la notion de fake news et son intégration dans une construction catholique de la vérité dans le discours du Pape François en 2018, avant que de conclure sur le rapport de la religion catholique (sinon aussi du journalisme) à la « vérité », ainsi posée.

\section{1. \\ Une opportunité d'affirmer un rapport singulier à la vérité}

Mais pourquoi donc l'Église catholique parle-t-elle, d'une façon qui peut somme toute paraître assez paradoxale, pour une institution porteuse de tant de récits, des fake news ? L'irruption sociale et médiatique du discours sur les fake news (Vanbremeersch, 2018) a sans doute constitué l'occasion d'un

2 Projet ANR Jeunes chercheurs "Vijie ", Vérification de l'information dans le journalisme, sur internet et dans l'espace public, enjeux sociétaux des dispositifs et outils numériques de lutte contre les "fausses informations ", conduit par Jérémie Nicey (Prim, Université de Tours, 2018-2021). 
positionnement institutionnel de " discours de la vérité " porté par une institution religieuse qui se vit comme porteuse déléguée d'un tel discours, l'Église catholique. Soucieuse de reprendre place dans un univers médiatique qu'elle ne contrôle pas (Dagenais, 1996), malgré le fait qu'elle dispose de ses propres médias (Anzelmo, 2018), l'Église catholique s'est en effet emparée à plusieurs reprises et par plusieurs de ses instances de la question des fake news, jouant de ce que nous pourrions penser être une simple équivoque, au motif qu'elle parle au nom de la " vérité ». En effet, dans l'institution de la mémoire (anamnèse) de son "fondateur " qu'elle entend former par ses rites, elle n'oublie pas que Jésus, sa référence principielle, a dit "Je suis le chemin la vérité et la vie » (Jn, 14, 16, cité par exemple par le Pape François en 2018 dans son message pour la journée mondiale des communications sociales) ni que le même évangile annonce que "La vérité vous rendra libres ! » $(J n$ 8, 32). Ceci fait de la proposition chrétienne une (pro)position qui se pense comme émancipatrice. Pour l'Église catholique, en effet, "Jésus [...] est la Vérité » (Viganò, 2017 ; Viganò, dans Menichetti 2018).

La fake news est en régime catholique pensée comme un obstacle à la communion humaine; elle altère la relation de communication. L'éradiquer relève donc pour ces chrétiens d'un combat théologique et spirituel autant que social, d'un rétablissement de la communication humaine (Pape François, 2017). La diffusion des mimétiques fake news, perçue comme un « mal de notre temps ", est pensée par cette institution comme l'occasion d'une diffusion de la vérité. Autrement dit, la notion de nouvelles falsifiées et de propagation de fausses nouvelles constitue aussi l'occasion d'une apologétique religieuse renouvelée, dans laquelle l'institution religieuse (pro)pose son rapport à la vérité. Elle devient l'institution de la vérité, puisque ancrée sur "la vérité même " (Lacan, 1974). L'appropriation de la thématique des fake news entendues comme symptôme de la société contemporaine enrichit les raisons d'un militantisme institutionnel très actif d'ordinaire sur des questions plus ciblées, touchant la famille (La Manif pour tous, les Veilleurs), la maladie et la mort ou la sexualité (Blanc, 2018 ; Raison du Cleuziou, 2019).

Quel que soit le rapport spécifique de cette institution à la vérité scientifique (ce qu'elle propose théologiquement n'en relève évidemment pas, quoi qu'elle ait pu ou puisse encore en dire), il nous paraît en effet pertinent, pour saisir l'appropriation qu'elle effectue de cette notion informationnelle, de suivre un temps son argumentation, sa rhétorique et sa rationalité propre. Car pour être fondée sur une "Révélation " (vérité révélée), la tradition théologique catholique ne s'en appuie pas moins sur un régime d'argumentation (Libera, 2003) et mobilise la rationalité (comme en témoignent les bibliothèques entières d'ouvrages théologiques, ou les travaux, parmi d'autres, de Thomas d'Aquin ou d'Anselme de Cantorbéry, tels le Fides quaerens intellectum, la foi recherchant l'intelligence), fût-ce à partir de prémisses et de données scientifiquement erronées, et inscrites dans le champ de « la foi ». 
L'élaboration chrétienne catholique de la notion de "fake news » et son utilisation comme arrimage d'une position catholique avec la culture médiatique contemporaine attestent du développement étique (c'est-à-dire interne) de cette compréhension des "fake news », dans une perspective à la fois théologique, sociale et politique. Elle manifeste une " manière d'habiter par l'énonciation des territoires communicationnels et symboliques", tant "les discours à propos des fake news relève[ent] [...] de jeux et d'enjeux de positionnement, d'identité ou de postures prises en fonction d'imageries ou d'imaginaires du "vrai" [...] " (Bodin et Chambru, 2018). On voit donc se former une interprétation théologique catholique de la désinformation numérique ou médiatique et de la falsification de l'information à des fins politiques. Cette prise de position sur les fake news s'inscrit bien entendu dans une tradition de prise de parole pontificale sur le journalisme (Defois et Tincq, 1997), depuis Pie XII et Paul $\mathrm{VI}$ - pour lequel la communication comme l'information doivent être régies par la vérité (Pape Paul VI, 1972) - au moins. Jusqu'au Pape François qui vise à suggérer l'honnêteté dans le traitement de l'information, celle-ci concourant à ses yeux à la paix : "le journaliste, gardien des nouvelles ", écrit ce dernier, " n'exerce pas seulement un métier, mais une véritable mission » dans le monde contemporain (Pape François, 2018).

\section{2. Des discours pontificaux prescriptifs à l'égard du journalisme}

Pour surprenant qu'il puisse paraître dans la théologie de l'information qu'il développe, en quelque sorte, ce discours du Pape François s'inscrit pourtant, en ne la renouvelant que d'un terme, dans une tradition d'allocutions d'enseignement pontifical à propos du journalisme et des médias qui, une fois que ces derniers ont été admis dans leur fonction sociale par l'Église catholique, visent à réguler leur action. Nous proposons de l'examiner ici - jusqu'en 1990 - à travers le recueil anthologique de textes consacrés aux médias et à la communication élaboré par le Groupe Médiathec $(1990)^{3}$ puis par les messages pour les journées mondiales de la communication sociale des papes tels que rassemblés aujourd'hui sur le site web du Vatican.

3 Par souci de brièveté dans la mention des références dans un article au format relativement restreint tel que celui-ci, nous ne pouvons malheureusement pas indiquer ici le titre et la nature exacts des textes cités, rassemblés dans I'anthologie française de référence publiée par le Groupe Médiathec, Les Médias, Textes des Églises (Centurion, 1990), et publiés dans l'organe officiel La Documentation catholique. Nous invitons donc les lecteurs à se référer à ce volume pour davantage de précisions contextuelles, si besoin. 
Une première tendance dans l'appropriation catholique des médias vient encourager et renforcer le journalisme chrétien. Ainsi pour la cardinal Pacelli (1931), futur Pie XII, « le journal catholique surgit providentiellement comme un phare de lumière chrétienne pour éclairer tous les faits et problèmes de la vie ${ }^{4}$. À la même époque (1933), pour le Pape Pie XI, les journalistes catholiques apparaissent, "pour user de la terminologie moderne ", comme "non plus seulement [des] porte-voix, mais [des] hauts parleurs de l'Église, de la vérité, de la foi [...] " ${ }^{5}$. Pie XI, dans une allocution à des journalistes romains, le 10 juin 1934, évoque les «formidables responsabilités [qui] sont inévitablement le lot des journalistes » car leur activité est " consacrée au service de la vérité et de la vertu ». Et de relever que "de salutaires effets » en découlent, inestimables : " personne ne pourra évaluer ce bien splendide, ce sillon lumineux dont bénéficient tant et tant d'âmes et d'intelligences ${ }^{6}$. On voit que la doctrine catholique sur la responsabilité véridictive des journalistes n'a pas profondément varié. Les dangers d'une infraction à ce service journalistique de la vérité sont soulignés par le pontife : «[...] si la parole, si l'écrit ne sont pas toujours ni tout entier au service de la vérité, des vertus et du bien des âmes, ils encourent une très grave responsabilité, car ils ouvrent la voie à un vaste mal d'une immense partie dévastatrice dont on ne peut calculer ni les méfaits ni les victimes ${ }^{7}$. Les journalistes, "maîtres " et " grands seigneurs de la parole ", mais aussi "radiateurs [sic] des splendeurs du bien et de la vie chrétienne », ont donc le devoir, poursuit le pape, citant alors le poète romantique italien Alessandro Manzoni (1785-1873), de " "ne jamais trahir la vérité sainte" ${ }^{8}$.

Après la seconde guerre mondiale, le rôle de l'information dans le conflit fait redire au Pape Pie XII (21 juillet 1945) les enjeux de la responsabilité du journaliste : «Un journaliste [...] conscient de sa haute vocation et de ses responsabilités, est toujours attentif à l'obligation qu'il a envers des milliers ou des millions de gens qui peuvent être fortement influencés par ses paroles, pour leur donner la vérité, rien que la vérité, autant qu'il a réussi à s'en assurer ${ }^{9}$. Et de renchérir, songeant peut-être aux accusations dont il fait l'objet, d'une façon qui annonce nos fake news : "Mais que dire du mensonge et de la calomnie délibérée? Le Seigneur hait une langue qui ment autant que des mains qui versent le sang innocent. Et tout homme juste déteste la parole mensongère (Prov. 6,$17 ; 13,5)$. La calomnie court vite, comme vous le savez [...] ${ }^{10}$. Les effets sociaux de l'absence de vérité dans l'information sont donc soulignés. Le pape, fortement controversé quant à son attitude durant la Seconde Guerre

4 Médiathec, 1990, p. 57.

5 Médiathec, p. 58-59.

6 Médiathec, p. 67-68.

7 Médiathec, p. 67-68.

8 Médiathec, p. 67-68.

9 Médiathec, p. 91.

10 Médiathec, p. 91. 
mondiale, poursuit en 1946 sa réflexion sur le journalisme et la vérité, devant des journalistes américains, en distinguant « la vérité de vision " ("c'est-à-dire que vous devez voir les événements tels qu'ils arrivent ") et la "vérité de présentation " ("vous devez rapporter les événements tels que vous les avez vu se produire et ne les interpréter que suivant les principes de la justice et de la charité »). Il dresse alors le portrait de la vérité : elle est " exempte de passion ", non partisane, elle "s'en tient à la réalité et non à l'imagination », elle " ne doit pas craindre d'être connue ", objective, "sans aucune influence de préjugés et de suppositions", elle est enfin discrète et modeste" ${ }^{11}$. Existe-t-elle ? Cela ne fait, en régime d'ontologie réaliste (les choses existent et l'esprit peut les connaître), aucun doute pour l'énonciateur. Le discours est le même, voire se fait plus grave en 1950 (23 janvier), devant un public semblable : «Qui ne sait qu'un journaliste peut délibérément falsifier des faits, ou, en les détachant de leur contexte, peut en déformer la véritable signification, ou peut supprimer la vérité qui réclame son droit d'être entendue ? Et le résultat net est que les masses sont égarées, qu'une tragédie humaine s'ensuit, que les conflits civils et même les guerres éclatent simplement parce qu'un membre indigne de votre profession, pour une raison ou une autre, a manqué à sa grave responsabilité envers la vérité ${ }^{12}$. Question d'équilibre du monde, donc, que la préoccupation de la vérité. Par conséquent, «La qualité principale du journaliste reste toujours un amour incorruptible de la vérité » (Pie XII, 1953) $)^{13}$.

Le Pape Jean XXIII, s'adressant à des journalistes catholiques italiens, dans leur congrès de 1959, poursuit le même propos : "Journalistes, vous devez d'une manière tout à fait particulière, par conscience professionnelle, chercher la vérité, afin que, si souvent foulée aux pieds et trahie par les moyens $d^{\prime}$ information, elle puisse triompher $! »^{14}$. De la radio, le pape dira précisément qu'elle est " une invitation à respecter la vérité », et qu'elle " engag[e] à dire la vérité, à vivre en elle » (12 février 1961) ${ }^{15}$. Les médias sont donc pensés comme subordonnés à la vérité, avec laquelle, comme le rappelle Ina Dvertanz (2016), I'Église catholique entretient un rapport particulier, de co-naturalité. Le discours du pape prononcé à l'adresse des journalistes accrédités à l'ouverture du concile Vatican II, le 13 octobre 1962, prolonge cette adresse : "Vous êtes au service de la vérité, et c'est dans la mesure où vous lui êtes fidèles que vous répondez à l'attente des hommes ${ }^{16}$.

\footnotetext{
11 Médiathec, p. 96.

12 Médiathec, p. 107.

13 Médiathec, p. 119.

14 Médiathec, p. 170.

15 Médiathec, p. 190.

16 Médiathec, p. 207.
} 
La production vaticane péri et post-conciliaire ${ }^{17}$ autour des médias et du journalisme semble moins accorder d'importance à la notion de vérité, qui disparaît au profit d'une attention portée, à propos des médias, à l'écoute de différentes tendances, au lien entre information et droits de l'homme, à la paix, à la lutte contre l'hégémonie idéologique ou économique, à la manipulation de l'opinion, aux dominations que les médias pourraient exercer; une attention aux voix inentendues paraît. Le dispositif médiatique semble plus d'écoute du monde que de relais de vérité ; il contribue néanmoins, comme pour le théologien brésilien Carlos Josaphat Pinto De Oliveira (Douyère, 2014) à la construction du monde en vue de l'advenue du « royaume de Dieu ». Néanmoins persiste la préconisation de l'objectivité faite aux journalistes.

Le Pape Paul VI cependant accorde de l'importance à la position journalistique et à la notion de vérité, qu'il reprend dans un discours adressé à la rédaction de L'Italia, en mai 1964 : " Le journal est un miroir et il doit être un miroir large et fidèle. Il obéit à une exigence fondamentale : celle d'informer, de rapporter les nouvelles, de dire les choses comme elles sont, de servir la vérité, cette vérité que nous pourrions appeler "photographique", la vérité des événements, des faits, de la chronique, la vérité sur le monde qui nous entoure et se meut autour de nous ${ }^{18}$. Le pape consacre d'ailleurs précisément son message pour la journée mondiale des communications sociales de 1972 à la question de la vérité et y invite à « saisir [...] la vérité entière [...] » $^{19}$.

Le thème de la Vérité revient avec la pensée de Jean-Paul II, qui est, pour celui-ci, à la fois " présentation de la réalité ", objectivité et indépendance ${ }^{20}$ et témoignage de la foi chrétienne (dans un sens institutionnel) au cours d'une allocution prononcée en 1983 pour le centième anniversaire du journal La Croix. À Los Angeles, en 1987, il s'adresse à des acteurs du monde médiatique en indiquant que " L'exercice correct du droit à l'information exige que le contenu de ce qui est communiqué soit vrai [...] ». Car "Votre profession même vous invite à réfléchir sur cette obligation à l'égard de la vérité et de son caractère exhaustif. Cela comprend aussi l'obligation d'éviter toute manipulation de la vérité pour quelque raison que ce soit [...] $»^{21}$. Le Pape Jean-Paul II consacre également à la question de la vérité une part (point 3, "Médias et vérité ») du message de la $37^{e}$ journée mondiale des communications sociales (Pape Jean-Paul II, 2003) «Les moyens de communication sociale au service de la paix authentique à la lumière de Pacem in Terris » et rappelle le nécessaire " respect envers la vérité » contre " la propagande et [la] désinformation » qui seraient « au service d'intérêts restreints, ou de préjugés nationaux, ethniques,

17 Le concile Vatican II se tient à Rome de 1962 à 1965.

18 Médiathec, p. 223.

19 Médiathec, p. 270.

20 Médiathec, p. 349.

21 Médiathec, p. 397-398. 
raciaux, et religieux, ou de l'avidité matérielle et des idéologies mensongères de plusieurs types ». La prescription véridictive accompagne le discours moral, social et politique de l'institution, elle fait partie de son système énonciatif.

La question de la vérité médiatique apparaît de nouveau dans les messages de son successeur, le Pape Benoît XVI, notamment dans le message pour la $42^{\mathrm{e}}$ journée mondiale des communications sociales (Pape Benoît XVI, 2008) "Les médias : au carrefour entre rôle et service. Chercher la Vérité pour la partager ». Critiquant "le matérialisme et le relativisme éthique », celui-ci affirme en effet que " les médias peuvent et doivent [...] contribuer à faire connaître la vérité sur l'homme, en la défendant devant ceux qui tendent à la nier ou à la détruire [...] que la recherche et la présentation de la vérité sur l'homme constituent la vocation la plus haute de la communication sociale ». Ici, les médias sont pensés comme devant être au service de l'anthropologie chrétienne et du combat moral de l'Église catholique (contre la liberté de l'avortement, le mariage homosexuel, etc.). S'ouvrant avec l'idée que « L'homme a soif de vérité, il est à la recherche de la vérité [...] », le paragraphe 6 de ce message insiste sur la conception théologique de la Vérité, qui est le Christ, libérateur, en des termes proches de ceux du Pape François dans le message de 2018 que nous étudions infra. Le Pape François évoque lui-même l'importance " d'aimer la vérité, une chose fondamentale pour tout le monde, mais spécialement pour les journalistes ", dans une allocution au Conseil italien de l'Ordre des journalistes, au Vatican, en 2016 (Pape François, 2017, 202). Il s'agit par là, pour le journaliste, de « vivre la vérité et $\left[d^{\prime}\right]$ en témoigner par son travail ».

\section{3. \\ La notion de fake news arrimée à une construction catholique de vérité}

Le message du Pape François "La vérité vous rendra libres, Fake news et journalisme de paix » a été diffusé pour la $52^{\mathrm{e}}$ journée mondiale des communications sociales ${ }^{22}$ (publié le 24 janvier 2018, en la fête de saint François de Sales, et prononcé au jour dédié, entre les fêtes catholiques de la Pentecôte et de l'Ascension). II reprend l'injonction de vérité adressée par les papes aux journalistes, mais en lui donnant un tour à la fois psychologique et théologique, et en la reliant à la perspective de la paix dans le monde. La question morale, dense pour ses deux prédécesseurs, semble disparaître. Le pape approche d'abord la question de la désinformation, dans sa dimension " mimétique »

22 Les messages pour les «journées mondiale des communications sociales» (Douyère, 2010) sont institués dans le prolongement du concile Vatican II dans la perspective d'une journée consacré aux médias et à la communication, associant réflexion, prière, mise en avant des acteurs de ce champ et quête dédiée. 
d'une information vraie (les fake news sont " plausibles » et "vraisemblables »), pour indiquer la façon dont elle vient flatter émotion et préjugés. Il loue les initiatives des acteurs du numérique et de l'éducation pour contrer ces « fausses nouvelles ».

Un changement de plan sémantique s'effectue dans le texte de la déclaration lorsque le pape catholique voit " "la logique du serpent" ", " capable partout de se dissimuler et de mordre " - soit la personne chrétienne et biblique du diable, cette instance qui divise et veut le mal, entend séparer l'Homme du dieu chrétien - derrière les fake news. Le " "père du mensonge" » utilise, dès la scène du jardin d'Éden, le mimétisme : son discours semble vrai (et incite Ėve à manger le fruit de l'arbre de la connaissance) mais ne l'est pas tout à fait ; il contrefait le vrai ; habile, il produit du semblant. Ceci constitue la « première "fake news" » (p. 3). Et le Pape François de conclure : « [...] aucune désinformation n'est inoffensive; de fait, se fier à ce qui est faux, produit des conséquences néfastes. Même une distorsion apparemment légère de la vérité peut avoir des effets dangereux " (p. 3). Il convient donc d'éviter de " mordre " [sic] à la tentation de la fausse information. La scène informationnelle sociale est ici lue, de façon très générale ${ }^{23}$, sur le plan d'un combat métaphysique réaliste, de la lutte entre le bien et le mal.

Les fake news sont ensuite reliées, de façon plus large, à une « contamination continuelle par un langage trompeur » (p. 3) qui atteint l'intériorité humaine. On passe ici, par une citation littéraire (Dostoïevski), au plan psychologique et moral, qui justifie si besoin la critique du faux. La vérité est définie dans une dimension existentielle comme " ce sur quoi l'on peut s'appuyer pour ne pas tomber » (p. 4). Le dieu chrétien, sous la figure de Jésus, apparaît bientôt : "Dans ce sens relationnel [de la vérité], le seul vraiment fiable et digne de confiance, sur lequel on peut compter, et qui est "vrai", est le Dieu vivant. Et c'est l'affirmation de Jésus : "Je suis la vérité" (Jn 14, 6) » (p. 4). Cette expérience de "fidélité et fiabilité de celui qui l'aime [...] libère l'homme : "La vérité vous rendra libres" $(J n$ 8, 32) » (p. 4). Le Christ est donc la vérité émancipatrice, tandis que les fake news sont une ruse du mal qui aliène. Une réflexion sur l'utilisation sociale d'une vérité, et sur ses effets pervers, conduit donc à la promotion de la

23 La dénonciation des fake news nous semble s'inscrire dans l'agenda médiatique, politique et social (Badouard, 2017, 39 et suivantes) du moment (influence désinformatrice dans le cadre de consultations nationales, aux USA, en France et au Royaume-Uni notamment) et ne nous semble pas viser certaines fausses nouvelles en particulier, même si l'on peut sans doute y entendre, pour des questions qui concernent plus spécifiquement l'Église catholique, la mobilisation de rumeurs dans la politique vaticane ou l'information sur des questions liées à la procréation et à la famille. Il ne semble pas que les propos du Pape François visent les informations concernant la pédophilie de clercs catholiques, question dont il s'empare par ailleurs. On trouve l'analyse d'un usage par des religieux de la dénonciation de supposées fake news dans l'article de Camila Arêas $(2018$ : 4, 20) consacré à l'Église universelle du royaume de Dieu s'implantant du Brésil au Portugal. 
personne " à l'écoute » et " sincère » (" meilleur antidote contre les faussetés ») comme élément essentiel de la construction de la vérité.

Un passage au plan d'une théologie des professions, pourrait-on dire, s'effectue alors : le journaliste, "gardien des nouvelles », exerce " une véritable mission ", celle de construire la paix par la vérité (et donc de lutter contre les fake news). Le Pape François promeut de la sorte « un journalisme sans duperies, hostile aux faussetés, aux slogans à effet et aux déclarations emphatiques [...], qui ne brûle ${ }^{24}$ pas les nouvelles, mais qui s'engage dans la recherche des véritables causes des conflits, pour en favoriser la compréhension à partir des racines [...]» (p. 5) ; un journalisme de "solutions alternatives », aussi. Pour mettre cela en pratique, le message s'achève par une prière, inspirée de celle attribuée à saint François d'Assise (référent symbolique chrétien du cardinal Jorge Mario Bergoglio, devenu le Pape François), adressée à " la Vérité en personne » (c'est-à-dire le Christ) et qui vise à une instrumentalisation positive, ancillaire, de "l'être informant ", comme disaient Palmer et Nicey (2010). II y s'agit, par un régime d'actions concret, de substituer à la rumeur, à la confusion, à l'ambiguïté, aux préjugés, à la fausseté, d'autres choses, parmi lesquelles la vérité, la clarté ou "les vraies questions », par exemple.

Le chemin discursif emprunté par cette déclaration pontificale est donc assez subtil, et ne se forme pas d'une opposition frontale entre le mensonge des fake news et la vérité du Christ, comme on aurait pu le supposer, mais s'appuie plutôt sur l'affirmation spirituelle - c'est-à-dire symbolique et langagière, mais aussi sociale (communautaire) - selon laquelle la relation à la personne du Christ permettrait d'entrer dans un processus qui éviterait la décomposition de la personne à laquelle conduiraient la diffusion et l'acceptation des fake news. Un processus social pacifiant devrait donc s'engager dans le traitement de l'information pour éviter cette diffusion de l'erreur trompeuse, et les journalistes en sont posés comme les acteurs majeurs.

Un commentaire de $M^{\text {gr }}$ Viganò (dans Menichetti, 2018), alors préfet du Secrétariat pour la communication du Vatican ${ }^{25}$ et auteur d'un ouvrage (Viganò, 2017) consacré à la communication "révolutionnaire " du Pape François - et qui place celle-ci, d'emblée, sous l'égide et au service de la vérité - précise le fonctionnement des fake news selon le pape : "Il est difficile de reconnaître des fake news parce qu'elles ont une physionomie mimétique : c'est la dynamique du mal qui se présente toujours comme un bien facilement accessible. L'efficacité dramatique de ce type de contenu consiste précisément à déguiser son propre mensonge, en semblant plausible pour certains [...] ». Le combat est donc tout à la fois métaphysique et informationnel.

24 Peut-être s'agit-il ici d'un jeu de mots avec l'expression « hot news », désignant l'information de dernière actualité.

25 Il démissionnera en 2018. 


\section{Conclusion}

Le rejet de la falsification propagandiste de l'information au motif que le Christ, à savoir, en théologie catholique, la seconde personne du dieu chrétien, " est Vérité ", n'est donc pas nouveau, et apparaît dès les déclarations pontificales des années 1930. II témoigne d'une culture religieuse marquée par un réalisme ontologique (Benoît XVI, 2009) qui lie fortement information et vérité (Douyère et Antoine, 2018, 65), d'une part, principe théologique et narration du monde, d'autre part. Cette dernière se doit d'être vraie, puisque le premier, selon elle, l'est. Cette articulation et cette injonction ne sont pas sans lien avec un certain positivisme journalistique (Ruellan, 1997), pour lequel le récit journalistique colle à la réalité et énonce la vérité des faits (Cornu, 1994). En régime catholique, cependant, une transcendance (autre qu'éthique) prévaut sur l'organisation informationnelle et l'Église est là, servante, pour inviter à la faire respecter. Plus encore, le trouble du monde se trouverait lié au mensonge. Ces déclarations témoignent donc d'une volonté de contrôle sur l'information et d'une exigence de vérité qui rejoint le mythe journalistique (Ruellan, 2007) lui-même, reconduit par le factchecking (Bigot, 2019) et la traque aux fake news (Vauchez, 2019), l'une et l'autre approche occultant la construction comme la subjectivité sinon la relativité même de l'information. On voit donc que le terme de "fake news " réactive et reconvoque une subordination de l'information à un principe théologique de vérité dans le discours catholique (Dufour, 2014), qui avait un peu disparu au moment des années 1960.

La fable réaliste, telle que narrée par le Pape François, du serpent auteur de fake news fait penser, en sens inverse, à celle de Jacques Derrida (2016) redessinant le sacrifice d'Isaac et faisant dire à Dieu, s'adressant à Jacob : "Surtout, pas de journalistes ! ". Ils seraient en effet capables à la fois de divulguer mais aussi sans doute de déformer la chose. Ne manipule pas les récits qui veut, pour cette (comme pour toute) institution - à l'heure même où les réseaux numériques offrent une grande plasticité narrative, et où la vérité, par-delà son lien avec le pouvoir, se trouve réduite à n'être qu'un désir. Comme l'écrit l'auteur, menteur repenti, Alexandre Jardin ${ }^{26}$ « le vrai est presque toujours absent du visible ». Mais la religion sait où le trouver. 
Bibliographie

Corpus et documents liés au terrain étudié

Benoît XVI, Pape (2009). Message urbi et orbi, Pâques. Disponible sur http://w2.vatican.va/content/ benedict-xvi/fr/messages/ urbi/documents/hf_ben-xvi_ mes_20090412_urbi-easter.html (page consultée le 1er juillet 2019).

Benoît XVI, Pape (2008). Les médias : au carrefour entre rôle et service. Chercher la Vérité pour la partager, message pour la $42^{\mathrm{e}}$ journée mondiale des communications sociales, 4 mai, Le Saint-Siège, Vatican. Disponible sur http://w2.vatican.va/ content/benedict-xvi/fr/messages/ communications/documents/hf_benxvi_mes_20080124_42nd-worId-communications-day.html (page

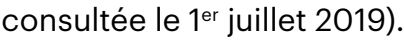

Bevilacqua A. (2018). « Le pape François s'élève contre les "fake news" ". In La Croix, 24/01. Disponible sur https://www.la-croix. com/Religion/Catholicisme/Pape/ Le-pape-Francois-seleve-contrefake-news-2018-01-24-1200908459 (page consultée le 1er juillet 2019).

François, Pape (2017), Politique et société, rencontres avec Dominique Wolton, Paris, Éditions de l'Observatoire.

François, Pape (2018). La vérité vous rendra libres, Fake news et journa- lisme de paix, message pour la $52^{\mathrm{e}}$ journée mondiale des communications sociales, 13 mai, Le Saint-Siège, Vatican. Disponible sur https://w2.vatican.va/content/francesco/fr/messages/communications/documents/ papa-francesco_20180124_messaggio-comunicazioni-sociali.html (page consultée le $1^{\text {er }}$ juillet 2019).

Jean Paul II, Pape (2003). Les moyens de communication sociale au service de la paix authentique à la lumière de Pacem in Terris, message de la XXXVII journée mondiale des communications sociales, $1^{\text {er }}$ juin, Le Saint-Siège, Vatican. Disponible sur http://w2.vatican.va/content/johnpaul-ii/fr/messages/communications/ documents/hf_jp-ii_mes_20030124_ world-communications-day.html (page consultée le 1er juillet 2019).

Médiathec, Groupe, Faculté de théologie de Lyon (dir.) [Bourgeois $\mathrm{H}$., Bianchi J., Kübler M., Keppel P., Buard J.-J.] (1990). Les Médias, textes des Églises, Paris, Le Centurion.

Menichetti M. (2018). « Les fake news, ennemi du journalisme de paix : réflexion de $\mathrm{M}^{\mathrm{gr}}$ Viganò, préfet du SPC ». In Vatican News, 24/01. Disponible sur https:// www.vaticannews.va/fr/vatican/ news/2018-01/message-pour.html (page consultée le 1er juillet 2019).

Paul VI, Pape (1972). « Les communications sociales au service de la vérité ", message pour la journée mondiale des communications sociales. In La Documentation catholique, vol. 1610 , p. 510-511. 
Turini N. [M"r] (dir.) (2017). « Église en réseaux. Quelle communion à l'ère du numérique ? ". In Documents Épiscopat, n 5, Paris, Conférence des évêques de France.

Viganò D. E. (2017). Frères et sœurs, bonsoir ! La communication du Pape François : une révolution, trad. D. Caldiroli, Montrouge, Bayard.

Références de recherche

Anzelmo A. (2018). "Les médias du Vatican face à aux questions féministe et de pédophilie ". In Revue française des sciences de l'information et de la communication, vol. 13. Disponible sur https:// journals.openedition.org/rfsic/3739 (page consultée le 1er juillet 2019).

Arêas C. (2018). « La communication religieuse en contexte de crise transnationale ". In Revue française des sciences de l'information et de la communication, vol. 13. Disponible sur : http://journals. openedition.org/rfsic/3693 (page consulté le 25 septembre 2019.

Badouard R. (2017). Le désenchantement de l'internet. Désinformation, rumeur et propagande, Limoges, FYP éditions.

Bigot L., (2019). Fact-checking vs fake news : vérifier pour mieux informer, Bry-sur-Marne, Ina éditions.

Bigot L., Nicey J. (à paraître). « Tous menteurs? Quand le fact-checking modifie les rapports du journalisme au politique ». In Decobert C., Rodriguez M.-S. (dir.), Construction et déconstruction du politique par les médias depuis 1975, Paris, Presses Sorbonne Nouvelle.

Blanc C. (2018). Théories et praxis de la "réinformation " : militantisme catholique traditionaliste et pratiques info-communicationnelles sur internet. Thèse de doctorat, Université Bordeaux Montaigne.

Bodin C., Chambru M. (2018). "Dossier thématique $\mathrm{n}^{\circ} 53$ "Fake news! Savoirs, pouvoirs et conflits dans l'espace public" ». Appel à articles, Études de communication. Disponible sur https://journals. openedition.org/edc/7790 (page consultée le 1er juillet 2019).

Cornu D. (1994). Journalisme et vérité. Pour une éthique de l'information, Genève, Labor \& Fides.

Dagenais B. (1996). " Les médias ont imposé une nouvelle logique à la religion ". In Communication et organisation, vol. 9, p. 73-108. doi: 10.4000/communicationorganisation.1840

Defois G., Tincq H. (1997). Les Médias et l'Église, Paris, CFPJ.

Derrida J. (2016). Surtout, pas de journalistes !, Paris, Galilée.

Douyère D. (2010). « La communication sociale : une perspective de l'Église catholique? Jean Devèze et la critique de la notion de "communication sociale" ". In Communiquer, vol. 3-4, p. 73-86. doi:10.4000/communiquer.1579 
Douyère D. (2014). « De la Propagande à une éthique de la communication sociale? L'approche politique et théologique du père $C$. J. Pinto de Oliveira ». In MEl, vol. 38, p. 79-90.

Douyère D. (2015). « Les mutations communicationnelles du christianisme catholique au $x x^{\mathrm{e}}$ siècle ". In Hermès, vol. 71, p. 225-235.

Douyère D. (2017). « $M^{\text {gr }}$ Norbert Turini (resp.), Église en réseaux. Quelle communion à l'ère du numérique?, Documents Épiscopat, $n^{\circ} 5,2017$, Paris, Conférence des évêques de France, 48 p. ". In Hermès, vol. 79, p. 229-235.

Douyère D. (2018). Communiquer la doctrine catholique. Textes et conversations durant le concile Vatican II d'après le journal d'Yves Congar, Genève, Labor \& Fides.

Douyère D., Antoine F. (2018). "Penser l'entrelacs des religions et des médias ». In Revue française des sciences de l'information et de la communication, $\mathrm{n}^{\circ} 13$. Disponible sur https://journals. openedition.org/rfsic/3756 (page consultée le 16 novembre 2019).

Dufour S. (2014), « La parresia : le Verbe et la vérité. L'évangélisation seIon le pape François ». In Bratosin S., Tudor M. A. (dir.), Espace public et communication de la foi. Les-Arcssur-Argens, larsic éditions, p. 137-149.

Dvertanz I. (2016), “Wahrheit und Kommunikation in der katholischen Kirche". In Wustmans C. (dir.),
Öffentlicher Raum. Theologische, religionswissenschaftliche und ethisch-normative Dimensionen, Hartmut Spenner, Kamen, p. 181-188.

Jeanneret Y. (2014). Critique de la trivialité. Les médiations de la communication, enjeu de pouvoir, Paris, Non Standard.

Lacan J. (1974). Le Triomphe de la religion. Précédé de Discours aux catholiques, Paris, Seuil.

Libera A. de (2003). Raison et Foi. Archéologie d'une crise, d'Albert le Grand à Jean-Paul II, Paris, Seuil.

Pace E. (2011). Religion as communication, God's Talk, Surrey (UK), Burlington (USA), Ashgate.

Palmer M., Nicey J. (2011). Lexique subjectif de l'homme informant, Paris, Éditions de l'Amandier.

Raison du Cleuziou Y. (2019). Une contre-révolution catholique, aux origines de la Manif pour tous, Paris, Le Seuil.

Ruellan D. (1997). Les " pro » du journalisme. De l'état au statut, la construction d'un espace professionnel, Rennes, PUR.

Ruellan D. (2007). Le journalisme ou le professionnalisme du flou, Grenoble, PUG.

Sauvageau P., Thibault S., Trudel P. (dir.) (2018). Les Fausses nouvelles, nouveaux visages, nouveaux défis. Comment déterminer 
la valeur de l'information dans les sociétés démocratiques, Québec, Presses de l'Université Laval.

Thibault S. (2018). "Craig Silverman : pionnier de l'enquête journalistique sur la désinformation en ligne ». In Sauvageau P., Thibault S., Trudel P. (dir.), Les Fausses nouvelles, nouveaux visages, nouveaux défis, Comment déterminer la valeur de l'information dans les sociétés démocratiques, Québec, Presses de l'Université Laval, p. 81-93.

Vauchez Y. (2019). « Les mythes professionnels des fact-checkeurs, un journalisme de données au service de la vérité ». In Politiques de communication, $n^{\circ} 12$, p. 21-44.

Vanbremeersch N. (2018). " De quoi les fake news sont-elles le nom?". In Le Débat, vol. 200, p. 15-22. 